\title{
Estudiando el fenómeno de la radiactividad a través de noticias de prensa: el caso del espía ruso envenenado
}

\author{
Soledad Esteban Santos y Javier Pérez-Esteban \\ Dpto. Química Orgánica y Bio-Orgánica - Facultad de Ciencias - UNED. España. \\ e mails:sesteban@ccia.uned.esyjpereze@ccia.uned.es
}

[Recibido en octubre de 2011, aceptado en enero de 2012]

\begin{abstract}
Los medios de comunicación constituyen una importante vía de divulgación científica. Pero es posible ir más allá, ya que mediante una estrategia apropiada ciertas noticias pueden convertirse en una herramienta didáctica para la educación formal de ciencias. En este sentido, se han seleccionado algunas notas de prensa que conllevan una importante carga de contenidos científicos relativos a la radiactividad, si bien hacen referencia a un hecho que se encuadra dentro de la crónica de sucesos. El objetivo es discutir algunas pautas metodológicas para emplear esas noticias en el aula de ciencias a fin de reforzar el estudio de una serie de conceptos y términos relativos al fenómeno de la radiactividad y hacer su aprendizaje más ameno y atractivo para los alumnos.
\end{abstract}

Palabras clave: radiactividad; educación formal; educación informal; medios de comunicación; herramienta didáctica; divulgación científica.

\section{Studying radioactivity through press news: the case of the Russian spy poisoning}

Media constitute an important way for scientific popularization. Nevertheless it is possible to go further, since some news can become an effective teaching aid in formal education of sciences by means of an adequate didactic strategy. In this sense, some pieces of news have been gathered due to their relation to scientific contents in the field of radioactivity, although the incident they referred to belongs to the section of accident and crime reports. The objective is to discuss some methodological guidelines to use that news in science classroom with the aim of reinforcing the study of important concepts and terms about the phenomenon of radioactivity, so that learning could become more interesting and attractive for students.

Keywords: radioactivity; teaching aid; formal education; informal education; media; scientific popularization.

\section{Introducción}

El proceso formativo de los individuos se puede articular desde distintos contextos. En primer lugar, se encuentran los contextos formales, totalmente estructurados y que se producen en instituciones educativas; después, los contextos no formales, estructurados sólo parcialmente y que tienen lugar fuera de las instituciones anteriores, aunque se produzcan en determinados recintos (que en lo que a ciencias se refiere serían, por ejemplo, museos y clubs de ciencias, exposiciones, talleres, etc.), y por último se cuenta también con los contextos informales, en los que están implicadas muy diversas situaciones pertenecientes más bien al ámbito cotidiano, como son prensa, películas, televisión, publicidad, Internet, etc. (De Pro, 2005), y en los que se puede aprender sin ni siquiera tener conciencia de que "se está aprendiendo".

Nos centraremos en este último caso, es decir, en los contextos informales, y concretamente en la formación en ciencias, para analizar algo más a fondo el aprendizaje que puede emanar de aquéllos. Estos contextos están totalmente desestructurados, no son secuenciados ni planificados y se centran en el sujeto que aprende, quien también va construyendo su propio aprendizaje, por lo que a veces pueden ser de poca utilidad y tener, incluso, efectos no deseados (por ejemplo, llegar a concepciones erróneas) Por otra parte, el aprendizaje informal es de carácter voluntario y fortuito, de libre elección, mientras que el formal se halla enmarcado en una estructura obligatoria y planificada, dirigido -o mejor, controlado- por el 
profesor, resultando al final de dicho aprendizaje formal una certificación emitida por la institución, una vez superadas las correspondientes pruebas de evaluación (Wellington, 1991; Rennie, Fecher, Dierking y Ellenbogen, 2003). En definitiva, el aprendizaje informal es muy distinto del formal, difiriendo además de este último en que se focaliza mucho más en aspectos sociales del conocimiento científico, lo cual promueve aún más un aprendizaje en contextos CTS, que acercan la ciencia a la vida cotidiana y mueven a la reflexión sobre su significado (Furió y Vilches, 1997; Gil y Vilches, 2001; Oliva, 2008).

Con todo lo anterior, los contextos informales parecen más bien encaminados hacia la divulgación científica y no a lo que entendemos, en sentido oficial, por educación en ciencias (Guerrero, 2002). No obstante, en el presente artículo nos planteamos la posibilidad de emplear los citados contextos con esta última finalidad. Siguiendo a Blanco (2004), entenderíamos como divulgación una recontextualización de ciertos conocimientos que se han adquirido previamente en contextos formales. Entonces, nos propondríamos lo que podría considerarse como un proceso inverso. Es decir, emplear los medios propios de la divulgación como recurso didáctico de la enseñanza formal, dentro de la escuela, para reforzar lo ya aprendido en ella y no sólo para recontextualizarlo. Este es bien conocido por todos los educadores, ya que son muy numerosos los estudios que se han llevado a cabo sobre su utilización en el aula (Millar y Osborne, 1998), diseñando propuestas para ello (Dimopoulos y Kouladis, 2005; Jarman y McClune, 2007) o haciendo hincapié en las dificultades inherentes a su puesta en práctica (Jiménez-Liso, Hernández-Villalobos y Lapetina, 2010).

Dentro de los contextos de aprendizaje informal, una interesante vía la constituyen los medios de comunicación y, sobre todo, la prensa. Muchos diarios incluyen semanalmente una sección dedicada a temas científicos, o bien aparecen esporádicamente artículos sobre algún problema o novedad científica. Por otra parte, hay que tener en cuenta que muy frecuentemente aparecen también artículos y noticias relacionados con aspectos científicos, si bien no tan explícitamente, sino de forma más bien indirecta (Oliveras y Sanmartí, 2008). Tal vez sea este último caso, el de artículos con contenidos científicos "encubiertos", el de mayor interés desde una perspectiva didáctica, ya que pueden resultar más atractivos para los alumnos. Y también para los profesores, ya que estimularían su imaginación para ir descubriendo poco a poco la forma de trabajar con esos artículos en el aula de la manera más eficaz, constituyendo así un reto que deberán superar. Porque hay que tener en cuenta, ante todo, el hecho de que para que se conviertan en una herramienta didáctica deben ser trabajados previamente por el docente, de forma reflexiva y profunda, a fin de extraer al máximo su potencial educativo.

En este sentido, discutiremos una noticia de este tipo que, aunque no se enmarque en los espacios habituales de la prensa dedicados a la ciencia, conlleva una significativa carga científica. Asimismo, se propone un itinerario didáctico a seguir en el aula, mediante el cual esa noticia podría convertirse en un recurso eficaz para revisar determinados contenidos científicos. Concretamente, en el caso que aquí trataremos esos contenidos son los correspondientes al fenómeno de la radiactividad y a otros contenidos relacionados.

El objetivo aquí perseguido es, pues, proponer unas pautas metodológicas para trabajar en el aula contenidos científicos relacionados con la radiactividad a través de notas de prensa sobre un hecho real. Esta propuesta didáctica no sólo resultaría enmarcada dentro del enfoque CTS, sino que además estaría de acuerdo con las ideas de Lemke en cuanto a rediseñar la educación científica acercando la ciencia a la realidad y proporcionando una imagen "honesta" de aquélla al mostrar tanto sus aspectos beneficiosos como los perjudiciales (Lemke, 1997 y 2006). 
El itinerario propuesto resultaría apropiado sobre todo para un primer curso de química de estudios universitarios de ciencias e ingenierías, para algunas asignaturas de bachillerato, como son las de química, e, incluso, la de Ciencias para el Mundo Contemporáneo. Pero también daría lugar a revisar contenidos de carácter más básico, como son los de átomo, núcleo atómico y partículas subatómicas, por lo que podría hacerse extensivo a otras asignaturas de ciencias de educación secundaria obligatoria.

\section{Punto de partida}

La noticia que hemos seleccionado trata de un hecho que tuvo lugar en los días de noviembre de 2006, extraño, casi insólito, lleno de interrogantes desde el punto de vista policial, pero también desde el punto de vista científico, que es el que aquí nos interesa. El protagonista fue Alexis Litvinenko, ex espía ruso que había pertenecido en su día a la KGB, exiliado en el Reino Unido y que vivía en Londres. En los momentos de este suceso estaba investigando sobre unos asuntos más o menos turbios relacionados con la guerra de Chechenia.

Litvinenko cayó enfermo el 1 de noviembre, después de una serie de reuniones con contactos rusos e italianos en la capital británica, y murió el 23 de ese mes, cuando sólo tenía 43 años, tras una agonía de casi cuatro semanas provocada por un altísimo nivel de radiación. Todavía en vida, los análisis de orina revelaron la presencia de un material radiactivo que, en principio, se pensó que era el talio. Pero el resultado de la autopsia reveló trazas de polonio 210 en su cuerpo. Además, su cadáver mostró dos focos de radiación, lo que indicaba dos envenenamientos con polonio en distintos días. Parece ser que la primera vez, se encontraba en una taza de té que tomó en el bar del hotel Millenium, en un lujoso barrio londinense. Y, efectivamente, ocho camareros resultaron contaminados también con polonio. El segundo envenenamiento pudo haberse producido días después en un restaurante japonés, mezclado en el "sushi". Se supone que fue envenenado porque sabía, o quería saber, demasiado.

No hay más que examinar los periódicos de esas fechas, yendo a una hemeroteca o, simplemente, acudiendo a Internet para revisar la versión digital de los diarios de mayor tirada.

Veamos algunos ejemplos en los fragmentos de algunos artículos periodísticos de esos días relativos a este suceso. Ante todo habrá que llevarse a cabo un análisis de los contenidos científicos que soportan, para lo cual se han resaltado en color los puntos que resultan esenciales para el objetivo que aquí perseguimos.

I. (El País Internacional, 24/11/2006)

Un análisis de orina apunta a que Litvinenko murió envenenado con
polonio radiactivo
El ex espía ruso hizo responsable de su muerte al presidente Putin en una
carta póstuma
...Las autoridades británicas cada vez tienen menos dudas de que el ex espía ruso Alexander
Litvinenko, conocido por sus feroces críticas al presidente ruso, Vladímir Putin, murió
envenenado por un agente químico altamente radiactivo, el polonio 210. Así lo ha anunciado en
rueda de prensa Roger Cox, de la Agencia de Protección de la Salud del Reino Unido, tras
analizar los restos de orina de Litvinenko, ex coronel de los servicios secretos soviéticos. Este
responsable médico ha dicho que en la orina se encontró una "gran cantidad" de radiación alfa
probablemente emitida por el polonio 210 . En paralelo a este anuncio, la policía británica ha
confirmado el hallazgo de un veneno radiactivo, cuyo nombre no ha revelado, en el restaurante
japonés de Londres donde cenó Litvinenko justo antes de caer enfermo...


II. (El Mundo, 30/11/2006)

La radiación del polonio 210 produce una muerte lenta y dolorosa
- El polonio 210 está disponible en la naturaleza y es muy difícil de manejar
- Se emplea en centrales nucleares y centros de investigación
... El envenenamiento de Alexander Litvinenko con polonio 210 ha despertado las dudas sobre
un elemento químico poco conocido fuera del ámbito de la energía nuclear... Se han registrado
18 internados por el caso del ex espía ruso y 12 sitios con radiación...

Incluso, en periódicos de otro tipo, por ejemplo de carácter deportivo, aparecieron referencias a este caso:

$$
\text { III. (Marca 05/12/2006) }
$$

\section{COMPATRIOTAS DEL FALLECIDO LITVINENKO ASISTIERON AL ARSENAL-CSKA DE MOSCÚ}

\section{Detectan rastros de polonio 210 en el estadio del Arsenal}

...Los investigadores británicos han hallado rastros de polonio 210 en el estadio londinense del Arsenal. La Agencia de Protección de la Salud (HPA) asegura que las cantidades de esta sustancia radiactiva son minimas y "casi imposibles de detectar"... La inspección del estadio del Arsenal inglés se llevó a cabo en el marco de la investigación de la muerte del ex espía ruso Alexander Litvinenko el pasado 23 de noviembre a causa de una alta dosis de polonio 210, ya que el pasado 1 de noviembre supuestamente asistieron dos compatriotas rusos de Litvinenko al partido Arsenal-CSKA Moscú de la Liga de Campeones después de haberse entrevistado con el ex agente secreto...

Estos artículos pueden clasificarse dentro de la crónica de sucesos, por lo que no se ubicarían en la sección que muchos periódicos dedican a la ciencia y la tecnología. Sin embargo, si se examinan más a fondo surgen preguntas directamente relacionadas con contenidos científicos. El punto de partida sería que el polonio se ha empleado como veneno, resultando además muy eficaz como tal, como puede observarse en la profunda transformación que en muy pocos días provocó en el protagonista de esta triste historia y que terminó en su trágica muerte (Figura 1).

Pero, ¿por qué es tan peligroso el polonio? Ésta sería la idea central de nuestro trabajo, alrededor de la cual fundamentaremos la estrategia seguida.
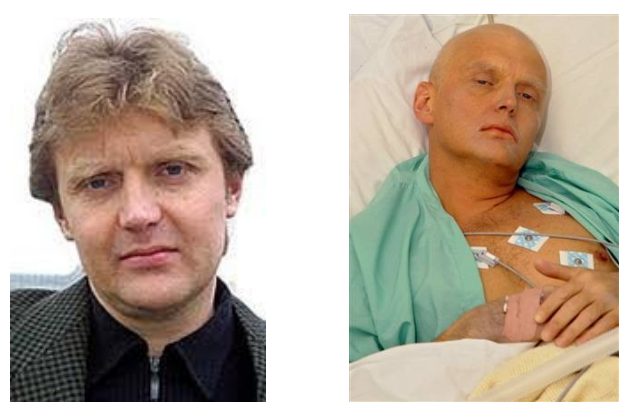

Figura 1. Litvinov antes y durante su enfermedad (envenenamiento con polonio).

A modo de ejemplo, proponemos a continuación un posible tratamiento en el aula a base de formularnos una serie de preguntas $y$, tras buscar las respuestas correspondientes, formar una 
trama de preguntas-respuestas para revisar determinados conceptos de química, algunos de ellos de carácter básico, y reforzar así su aprendizaje.

\section{Metodología}

Ante todo el docente habrá de plantearse qué pretende conseguir con el tratamiento en el aula de noticias de este tipo. Ello exige que previamente, fuera del aula, lleve a cabo un análisis de sus contenidos realizando un minucioso examen de esas noticias para detectar qué aspectos relacionados con la ciencia incluyen. Este examen delimitará los objetivos didácticos a alcanzar y le permitirá diseñar una trama de preguntas-respuestas, estructurándola en función de la asignatura y nivel de sus alumnos. Después, ya en el aula, se trabajará con ese diseño.

Siguiendo con nuestro caso, expondremos a modo de sugerencia y ejemplo el proceso seguido, teniendo en cuenta que nos centraremos en el problema radiactividad/salud, dejando aparte el de radiactividad/energía, a fin de no extendernos demasiado y acotar contenidos. Para hallar las respuestas a las preguntas de la trama se han empleado textos de Química de primer curso universitario.

\section{Análisis de contenidos}

Volvamos a la primera de las noticias aquí expuestas, analizando desde una perspectiva científica lo que se había remarcado en color:

... Alexander Litvinenko... murió envenenado por un agente químico altamente radiactivo, el polonio $210 \ldots$ en la orina se encontró una "gran cantidad" de radiación alfa probablemente emitida por el polonio $210 \ldots$ veneno radiactivo...

En definitiva, tenemos que Litvinenko murió envenenado por polonio 210, agente químico muy radiactivo, y que se encontró en su orina radiación alfa emitida probablemente por el polonio.

Fijémonos en algunos de esos términos, aparentemente inconexos:

$$
\text { Envenenado, veneno - Agente químico - Radiactivo - Polonio } 210 \text { - Radiación alfa }
$$

En primer lugar podemos hacernos estas dos preguntas:

- 1. ¿Qué es el "polonio”?

- 2. ¿Qué significa "radiactivo”?

Pero después podríamos plantearnos otras cuestiones:

- 3. ¿Por qué es radiactivo el polonio?

- 4. ¿Qué es una radiación alfa?

- 5. ¿Cuáles son los efectos de las radiaciones alfa?

- 6. ¿Qué tiene que ver el polonio con las radiaciones alfa?

- 7. ¿Por qué se escribe siempre polonio 210?

Y, volviendo a la idea central de nuestro trabajo, tendríamos:

- 8. ¿Por qué es el polonio tan peligroso que sirve para "envenenar" hasta producir la muerte?

Comencemos por las dos primeras. Ambas preguntas y sus respectivas respuestas constituyen las claves de las que surgirán de forma fluida y natural otra serie de preguntas, y también nos 
darán las respuestas de muchas de las otras cuestiones que inicialmente nos hemos formulado. Vertebrarían, pues, toda una trama de preguntas-respuestas.

\section{1. ¿Qué es el polonio?}

La respuesta inmediata podría ser decir, simplemente, que el polonio es un elemento químico.

De ahí, surgiría a su vez, la pregunta siguiente:

¿Qué es un elemento químico? $\boldsymbol{R}$ : Un elemento químico es una sustancia constituida por un único tipo de átomos.

Y de esta respuesta surgirían a su vez otra serie de cuestiones, de las cuales la primera podría ser:

¿Cómo se caracteriza un elemento químico determinado? $\boldsymbol{R}$ : Se caracteriza por lo que se conoce como número atómico.

Lo que nos llevaría a plantearnos una nueva cuestión:

¿Qué es el número atómico? $\boldsymbol{R}$ : Es el número de protones del núcleo de un átomo (también, número de electrones de un átomo eléctricamente neutro). O número de orden de dicho elemento en la Tabla Periódica.

En el caso del polonio, consultando la Tabla Periódica resulta que su número atómico es 84.

Todo esto, por su parte, podría llevarnos a revisar los conceptos de:

$>$ sustancia

$>$ átomo

> partículas fundamentales: protón, neutrón, electrón

$>$ constitución del átomo:

- núcleo: constituido por protones y neutrones $\rightarrow$ número atómico y número másico

- envoltura electrónica: constituida por electrones $\rightarrow$ configuración electrónica

- modelos atómicos (distintos modelos, discutidos en función del nivel de conocimientos previos del alumnado)

Si bien los conceptos básicos de sustancia, átomo, etc. podrían ser objeto de un tratamiento similar, aquí nos hemos centrado en otros más directamente implicados en el estudio de la radiactividad:

Número másico (o número de masa): número de protones más número de neutrones de un núcleo atómico.

Isótopo: átomos cuyo núcleo tienen el mismo número de protones, pero diferente número de neutrones (es decir, diferente número másico).

Se remarcaría que: a) el número atómico caracteriza a cada elemento químico y lo distingue de los demás, ya que los átomos de un determinado elemento tienen el mismo número atómico, y b) en los isótopos se conserva el número de protones (número atómico), por lo que se trata de átomos del mismo elemento, pero no se conserva el de neutrones. Por ello, 
cuando se hace referencia a un determinado isótopo de un elemento es necesario indicar su número másico.

Por tanto, en un mismo elemento se conserva el número atómico, pero en los isótopos no se conserva el número másico.

Con estas premisas, podremos seguir adelante con las cuestiones formuladas en un principio.

\section{2. ¿Qué significa "radiactivo"?}

La palabra "radiactivo" es un adjetivo concerniente al término radiactividad.

Aunque éste sea un término que resultará familiar a los alumnos (puesto que de una manera u otra todos hemos oído hablar de radiactividad), debemos profundizar en este fenómeno, formulando las siguientes cuestiones:

- ¿En qué consiste la radiactividad y cómo se manifiesta?

- ¿Por qué tiene lugar?

¿En qué consiste la radiactividad?

En que ciertos materiales espontáneamente emiten unas radiaciones de gran energía. En un principio este hecho se detectó en materiales que existían en la naturaleza (radiactividad natural). No obstante, con el tiempo se ha conseguido provocar o inducir procesos radiactivos, por lo que este fenómeno se produce de forma artificial (radiactividad artificial).

¿Por qué tiene lugar, es decir, por qué se producen esas radiaciones?

Para comprenderlo hay que analizar ante todo el núcleo de los átomos, en el que se sitúan protones y neutrones dentro de un espacio muy reducido, empaquetados unos junto a otros como en un cesto de naranjas. Entre los protones, partículas de carga eléctrica positiva, existirá una gran repulsión electrostática. Y para que el núcleo sea estable, deberá haber unas fuerzas que mantengan cohesionadas todas sus partículas, capaces de vencer esas enormes fuerzas de repulsión entre los protones. Efectivamente, existen también fuerzas de atracción protones-neutrones y neutrones-neutrones, que habrán de superar las repulsiones protónprotón.

Pero, ¿qué ocurriría si no las superaran? Obviamente que el núcleo sería inestable. Estos núcleos inestables o radiactivos sufren determinados cambios por los que se transforman en otros núcleos diferentes, proceso acompañado de la emisión de radiaciones de elevada energía. Se dice que sufren una desintegración y esta transformación es lo que se conoce como reacción nuclear.

La razón última de la radiactividad es, pues, la presencia de núcleos atómicos inestables, por lo que en las reacciones nucleares el número atómico no se mantiene.

¿Por qué unos núcleos son inestables y otros no?

En los elementos de las sustancias que nos rodean y con las que trabajamos habitualmente, los núcleos son estables. En la naturaleza hay elementos que tienen isótopos estables y también isótopos radiactivos, pero existen otros elementos en los que todos los isótopos son radiactivos. Se ha comprobado así que además del tecnecio $(Z=43)$ y del prometio $(Z=61)$ los núcleos atómicos con más de 83 protones (es decir, de número atómico superior a 83) son inestables, independientemente del número de neutrones presentes. Podría explicarse este fenómeno porque al haber una concentración de carga positiva tan grande en un espacio muy pequeño como es el núcleo atómico, las repulsiones electrostáticas resultarían tan enormes que 
no podrían ser compensadas por las fuerzas atractivas en las que los neutrones están implicados.

\section{3. ¿Por qué es radiactivo el polonio?}

Ahora ya tenemos respuesta a esta cuestión: porque el número atómico del polonio es 84 , obviamente superior a 83 .

\section{4. ¿Qué es una radiación alfa?}

Al desintegrarse los núcleos inestables o radiactivos emiten unas radiaciones, que son de distintos tipos. Las radiaciones más importantes son:

> Radiaciones alfa (a): consisten en una emisión de partículas alfa, partículas con carga positiva, idénticas a los núcleos de helio $\left({ }_{2}^{4} \mathrm{He}\right)$. La desintegración con emisión de partículas alfa generalmente tiene lugar sólo en elementos con número másico superior a 200.

> Radiaciones beta ( $\beta$ ): consisten en la emisión de partículas beta, de carga negativa y propiedades idénticas a los electrones de los orbitales, pero cuyo origen es diferente ya que provienen del núcleo.

> Radiaciones (o rayos) gamma ( $\gamma$ ): ya no son partículas sino radiaciones electromagnéticas de longitud de onda inferior a las de los rayos $\mathrm{X}$, siendo por ello su energía y poder de penetración mayores que las de éstos.

\section{5. ¿Cuáles son los efectos de las radiaciones alfa?}

Todas las radiaciones llevan asociada una energía, son ionizantes y poseen un poder de penetración, aspectos fundamentales a tener en cuenta respecto al daño que pueden provocar a los organismos vivos.

En cuanto a su poder de penetración, resulta que:

- las partículas alfa, al ser tan pesadas, tienen un poder de penetración limitado, pudiendo ser detenidas por unas hojas de papel, la ropa e, incluso, la propia piel.

- las partículas beta son ya más penetrantes que las alfa por tener una masa mucho menor y para detenerlas se necesita, por ejemplo, una lámina metálica de $3 \mathrm{~cm}$ de espesor.

- la radiación gamma, aunque muy poco ionizante, es la de mayor poder de penetración por no tener ni masa ni carga eléctrica, y puede atravesar completamente el cuerpo humano, y para detenerlas se requiere una gruesa capa de plomo o de cemento.

\section{Daños de las radiaciones a los sistemas biológicos}

Pero, ¿̇uál es la justificación, desde un punto de vista químico, de la producción de estos daños en el organismo? La causa es el carácter ionizante de las radiaciones. Por su elevada energía son capaces de arrancar electrones a los átomos y moléculas que se encuentran a su paso, formando así iones y radicales. Los radicales, llamados también radicales libres, son especies químicas con uno o más electrones desapareados. Este hecho les hace ser sumamente reactivos ya que reaccionan tratando de aparear esos electrones. Atacan así las membranas celulares de los tejidos, las funciones orgánicas de enzimas y $\mathrm{ADN}$, etc., con lo cual las biomoléculas terminan destruyéndose. 
Esto constituye una aparente paradoja:

Por una parte, los isótopos radiactivos son muy peligrosos para nuestro organismo. Pero, por otra, a veces ciertos isótopos radiactivos se emplean con fines terapéuticos porque pueden destruir células malignas: tal es el caso del cobalto o del radio en el tratamiento del cáncer (radioterapia), o del yodo radiactivo en tumores del tiroides.

Los daños causados en el organismo dependen de la cantidad de radiación absorbida, de la energía y del poder de penetración de la radiaciones (es decir, del tipo de radiación de que se trate). Pero también el daño dependerá de la región del cuerpo expuesta a la radiación. Por este motivo hay que introducir un factor que se conoce como efectividad biológica relativa.

Las radiaciones gamma son más penetrantes, seguidas de las partículas beta, mientras que las partículas alfa son las de menor poder de penetración. Sin embargo, aunque estas últimas sean las menos penetrantes, los daños que causan pueden llegar a ser muy graves según las circunstancias, debido a que los órganos estarán sometidos a una radiación constante de corto alcance. Se ha comprobado que la radiación alfa es mucho más perjudicial (unas 20 veces más).

La radiación alfa es relativamente inofensiva cuando es externa al cuerpo (por ejemplo, si tocamos un material que emite esta radiación), ya que es absorbida por la capa superficial de piel muerta; pero si se inhala o se ingiere es extremadamente peligrosa por la gran energía del impacto de unas partículas de masa tan considerable dentro del organismo, con lo cual inactivan las biomoléculas y pueden llevar, incluso, a la muerte.

Con todo esto, ya se ha dado la respuesta a las cuestiones 4 (¿qué es una radiación alfa?) y $\mathbf{5}$ (¿cuáles son sus efectos?). Y para completar nuestra serie inicial de preguntas, volveremos sobre el polonio para conocer algo más sobre el mismo.

\section{Algo más sobre el polonio}

Se puede ampliar la respuesta a la primera cuestión de qué es el polonio, y conocer algo más sobre este elemento. Para ello, podemos hacer una consulta en textos de Química o en Internet, por ejemplo, en Wikipedia:

El polonio es un elemento químico, de símbolo Po y de número atómico 84 y generalmente está presente en los minerales de uranio. Fue descubierto en 1898 en la pechblenda por Marie y Pierre Curie. De ahí su nombre, como homenaje al país natal de Marie. Todos sus isótopos son radiactivos.

Ya hemos sabido por qué es radiactivo, como todos los elementos a partir del bismuto (83).

De los 27 isótopos que se le conocen es el polonio 210 el único disponible en la naturaleza, aunque en muy pequeñas cantidades. Proviene de la desintegración del uranio 238, muy común, por ejemplo, en los suelos graníticos.

Ahora es posible dar respuesta a otra de nuestras cuestiones iniciales, la cuestión 7: ¿por qué en esos artículos se escribe siempre polonio 210? Como el polonio tiene muchos isótopos, para distinguirlos entre sí, se escribe el número másico. Por tanto, en estos artículos se trata del isótopo del polonio de número másico 210. Ese número de 210 hace referencia, pues, al número másico.

Este isótopo de polonio tiene un periodo de semidesintegración de 138,39 días y es un fuerte emisor de partículas alfa. Libera así gran cantidad de energía, por lo que 
se le ha utilizado como fuente energética en las células termoeléctricas de algunos satélites artificiales y de sondas lunares.

Como ya se dijo al tratar de las radiaciones alfa, generalmente sólo son producidas por los núcleos radiactivos de número másico superior a 200. Éste es el caso del polonio 210, lo que explica que produzca radiaciones de este tipo, lo cual da respuesta a la cuestión 6 (zqué tiene que ver el polonio con las radiaciones alfa?).

Por otra parte, las partículas alfa son muy dañinas para el organismo, lo que justifica la peligrosidad del polonio 210 (cuestión 8). Además en estas notas sobre el polonio se señala que este isótopo tiene un periodo de semidesintegración de 138,39 días. Teniendo en cuenta que el periodo de semidesintegración es el tiempo necesario para que desaparezca la mitad de la muestra radiactiva, emitirá radiaciones alfa durante bastante tiempo.

Ya hemos contestado así a la idea central de este suceso, la capacidad del polonio como agente venenoso tan eficaz.

Se puede ampliar aún algo más sobre las propiedades del polonio:

Observando la Tabla Periódica podremos situarlo en ella: sexto periodo y columna 18. Está pues muy próximo al bismuto y al telurio, por lo que se les parece mucho en sus propiedades, siendo como ellos un metaloide. Pero, se diferencia de ambos en que el polonio es radiactivo, mientras que los otros dos no lo son.

Podría aquí profundizarse en el concepto de metaloide o semiconductor, lo cual nos llevaría también a revisar el concepto de metal y no metal, las características generales de metales y no metales y, con ello, el concepto de electronegatividad. No obstante, esta derivación se haría si así lo juzgase oportuno el profesor.

\section{Interpretación cientifica de este suceso}

Podemos interpretar lo sucedido con Litvinenko desde el punto de vista químico, haciendo una síntesis de todo lo anterior:

El polonio es una sustancia sumamente peligrosa para los seres vivos: es radiactivo y emite gran cantidad de partículas alfa. Si recordamos, las partículas alfa son mucho más peligrosas cuando se introducen en el interior del organismo, como fue en este caso, ya que el polonio fue ingerido a través del té o en alguna comida. Además el periodo de semidesintegración del polonio es de 138,39 días: es decir, que la mitad de la muestra radiactiva tardaría esto en desparecer, quedando aún en el organismo restos de polonio durante bastante tiempo. Al permanecer en el interior del cuerpo, provoca la desintegración de las biomoléculas, produciendo daños en los tejidos y órganos vitales a veces irreparables.

Esto es lo que ocurrió con Litvinenko. En definitiva, no había solución para salvar su vida. Es toda una historia real que supera con mucho la ficción. Un clásico perfecto para una estupenda novela policíaca o para una película, como de hecho ocurrió en 2007 (Rebelión: el caso Litvinenko, dirigida por Andrei Nekrasov).

\section{Itinerario didáctico}

Con la base de esa trama preguntas-respuestas, se trabajará en el aula con los alumnos, fomentando su participación activa mediante distintas vías: haciendo que ellos mismos lleven a cabo la búsqueda de más noticias sobre este suceso, de información adicional sobre aspectos científicos, etc. (en libros de texto, Internet o hemerotecas) y promoviendo el debate sobre determinados problemas relacionados con la radiactividad. 
El itinerario didáctico podría ser la siguiente:

- Resolución por los alumnos de un breve cuestionario previo para detectar lo que conocen sobre la radiactividad, diseñado por el profesor (ver Anexo).

- Exposición por el profesor, a grandes rasgos, de este suceso.

- Búsqueda por parte del alumnado (individualmente o en grupos) a través de Internet de más noticias sobre este suceso aparecidas en distintos periódicos de la prensa digital $\mathrm{o}$, incluso, en hemerotecas

- Realización por los alumnos de la lectura de todas las noticias, primero de los titulares y después de los artículos completos

- Marcar las palabras que según el criterio del alumnado estarían relacionadas con la ciencia.

- Hacer una síntesis de esa lectura, llevada a cabo por los alumnos con la supervisión del profesor.

- Formular, siempre bajo la guía del profesor, una serie de preguntas y buscar sus respuestas, formando así una trama según las pautas anteriormente expuestas (se tratarían así en el aula aquellos contenidos científicos que a juicio del docente fueran los más adecuados, teniendo en cuenta como siempre la asignatura y el nivel del alumnado).

- Elaborar un glosario partiendo de los contenidos tratados en la secuencia preguntasrespuestas.

- Organizar un debate sobre algún aspecto de la radiactividad.

- Finalmente, se entregaría a los alumnos un segundo cuestionario, a fin de detectar lo que hayan aprendido sobre este tema (ver Anexo).

\section{Conclusiones}

Un hecho como éste, que invadió los distintos medios de comunicación hace unos años y acaparó por unos días la atención mundial, puede convertirse a través de una serie de estrategias en un eficaz recurso didáctico, ya que:

- Muestra a los estudiantes cómo la química no está tan lejos de la vida real, por extraña que ésta sea, con lo que abre paso a percibir las relaciones ciencia-sociedad.

- Dado el innegable sesgo de intriga de este suceso, puede ser un buen elemento de motivación hacia el estudio de la química en particular, y la ciencia en general, despertando la curiosidad por saber más.

- Proporciona una fluida secuencia de preguntas-respuestas para hacer una revisión, más o menos amplia -dependiendo del objetivo perseguido por el docente- de unos contenidos científicos determinados, muchos de ellos de carácter básico.

- Facilitar con todo ello que el alumnado consolide el aprendizaje de esos contenidos científicos.

- Permite enlazar con la historia de la ciencia: en este caso, se conectaría fácilmente con el descubrimiento de la radiactividad, sus actores (Henri Becquerel, Marie y Pierre Curie), el proceso seguido (resaltando, por ejemplo, con el carácter de descubrimiento fortuito, como tantos otros), etc. 
- Promueve el debate sobre la radiactividad, sus aplicaciones, peligros y ventajas, todo lo cual favorece el espíritu crítico y la adquisición de una actitud ética y reflexiva hacia la ciencia.

- Da lugar a que el alumnado adquiera otras competencias, como la de realizar una lectura crítica de los artículos de prensa y, en general, de cualquier texto científico.

- Aficiona a los alumnos por la lectura de la prensa, una de las principales fuentes de información.

\section{Referencias bibliográficas}

Blanco, A. (2002). Relaciones entre la educación científica y la divulgación de la Ciencia. Revista Eureka sobre Enseñanza y Divulgación de las Ciencias, 1(2). 70-86. En línea en http://reuredc.uca.es

De Pro, A. (2005). La enseñanza no formal de las ciencias, Alambique, 43, 6-7.

Dimopoulos, K. y Koulaidis, V. (2003). Science and Technology Education for Citizenship: The potential Role of the Press. Science Education, 87 (2), 241-256.

Furió, C., Vilches, A. (1997). La Enseñanza y el Aprendizaje de las Ciencias de la Naturaleza en la Educación Secundaria. Barcelona: Horsori.

Gil, D. y Vilches, A. (2001). Una alfabetización científica para el siglo XXI. Obstáculos y propuestas de actuación. Investigación en la Escuela, 43, 27-37.

Guerrero, R. (2002). La divulgación científica en el siglo XX: de Wells a Gould. Quark, 26. En línea en http://www.prbb.org/quark/26/Defaukt.htm

Jarman, R. y McClune, B. (2007). Developing scientific literacy. Using news media in the classroom. Maidenhead, Berkshire: Open University Press. McGraw-Hill.

Jiménez-Liso, M.R.; Hernández-Villalobos, L. y Lapetina, J. (2010). Dificultades y propuestas para utilizar las noticias científicas de la prensa en el aula de ciencias. Revista Eureka sobre Enseñanza y Divulgación de las Ciencias, 7(1). 107-126. En línea en http://reuredc.uca.es

Lemke, J.L. (1997). Aprender a hablar ciencia. Barcelona: Paidós.

Lemke, J.L. (2006). Investigar para el futuro de la educación científica: nuevas formas de aprender, nuevas formas de vivir. Enseñanza de las Ciencias, 24(1), 5-12

Millar, R., y Osborne, J. F. (Eds.). (1998). Beyond 2000: Science Education for the Future. London: King's College London.

Oliva, J.M. (2008). Editorial: Ciencia recreativa, educación científica en contextos y mediante recursos no formales, e investigación en Didáctica de las Ciencias. Revista Eureka sobre Enseñanza y Divulgación de las Ciencias, 1(1). 1. En línea en: http:// reuredc.uca.es

Oliveras, B. y Sanmartí, N. (2008). Análisis de una actividad orientada a promover la lectura crítica en las clases de Ciencias. En Jiménez-Liso, M.R. (Ed.). Ciencias para el Mundo Contemporáneo y Formación del Profesorado en Didáctica de las Ciencias Experimentales. Actas de los XXIII Encuentros de DCE, 149-159. En línea en: http://www.ual.es/Universidad/ Depar/dmce/Congreso/Actas\%2023EDCE.pdf

Rennie, L.; Fecher, E.; Dierking, L. y Falk, J. (2003). Towards an agenda for advancing research on science learning in out-of-school settings. Journal of Research in Science Teaching, 40(2), 112-120.

Wellington, J. (1991), Newspaper science, school science: friends or enemies? International Journal of Science Education, 75 (1), 9-21. 


\section{ANEXO}

\section{Cuestionario Inicial}

Por favor, responde brevemente a las siguientes cuestiones.

1. ¿Has oído hablar de la radiactividad? En caso afirmativo, enumera las ocasiones que recuerdes.

2. ¿Piensas que es algo negativo?

3. ¿Podrías indicar algunas de sus aplicaciones?

\section{Cuestionario Final}

Por favor, responde a las siguientes cuestiones, dando una breve explicación a las mismas.

1. ¿En qué consiste la radiactividad y cómo se manifiesta?

2. ¿Piensas que éste es un fenómeno sólo natural? ¿Por qué?

3. ¿Asocias este fenómeno a algo negativo? Justifica tu respuesta brevemente.

4. ¿Piensas que pueda tener alguna aplicación práctica positiva? Justifica tu respuesta brevemente. 